C O M M E N T A R I E S
The Architecture of Connective Tissue
in the Musculoskeletal System-Often
Overlooked Functional Parameter as to the
Proprioception of the Locomotor Apparatus

\section{Original Citation}

Van der Wal, JC. The architecture of connective tissue in the musculoskeletal system-often overlooked functional parameter as to the proprioception of the locomotor apparatus. IJTMB. 2009;2(4):9-23.

In December 2009 (IJTMB Volume 2, Number 4), an article was published, titled The Architecture of Connective Tissue in the Musculoskeletal System-Often Overlooked Functional Parameter as to the Proprioception of the Locomotor Apparatus. Published online 2009 Dec 7. doi: 10.3822/ijtmb.v2i4.62

In this manuscript, it was proposed that traditional dissection anatomy is not the right tool to describe the functional organization of fascia and connective tissue, since the fascia and fascial structures are not about topography or anatomy, but may differ substantially in their architectural and 'mechanical' relationship with the muscle and bone tissue. This concept has major implications for understanding the organization of the substrate through which proprioception is communicated in the so-called musculoskeletal system. This organization cannot be described in terms of the usual discrete elements like muscles, bones, ligaments, tendons, and so on, but has to be described in terms of functional architecture relationships that this substrate (mechanoreceptors) has with fascia. Initially presented at the Second International Congress on Fascia Research (2009), the published paper was welcomed as an important shift in thinking about 'fascial anatomy'.

Although the article has been quoted many times, it was not until January 2020 that some quite essential errors in the figures' captions were brought to the author's attention by Prof. $\mathrm{H}$. van Mameren, under whose supervision the research that the article is based upon was carried out. So, in good deliberation with the IJTMB editors, this commentary serves to correct the errors, and to honor belatedly the copyright of the figures as claimed by the rightholder of that time.

\section{Corrected Captions (corrections in bold)}

FIGURE 7. Opening of the antebrachial fascia including retinaculum extensorum in the distal dorsolateral region. Intermuscular loose areolar connective tissue revealed between the discrete muscle bellies and tendons. Left arm, dorsal side, lateral view.

FIGURE 2. The compartment of the third extensor digitorum muscle is opened and partly separated from the muscle fibers. Proximally muscle fibers are still inserted to compartment walls. Left arm, dorsal side, lateral view.

FIGURE 3. Proximal medial elbow region. Muscles are dissected away from the epicondylar connective tissue apparatus and reflected (to the right). Also, on the medial elbow region, muscle compartment walls converge toward the humeral epicondyle. Left elbow, medial view, image left-right mirrored.

FIGURE 4. Proximal medial forearm region. Muscles and muscular tissue have been removed. The most proximal extensions of the muscle compartment walls (the epicondylar connective tissue apparatus) are left in situ, demonstrating the muscle compartments converging to the medial epicondyle. Left elbow, medial view, image left-right mirrored.

FIGURE 5. (a) The "classical" in-parallel organization of the iuxta-articular tissue. From inside to outside: articular capsule (blue); reinforcing iuxta-articular regular dense connective tissue structures (ligaments) (yellow); and on the outer side, periarticular muscle (red). (b) The "classical" organization principle of iuxta-articular connective tissue running from bone to bone, organized in parallel to the muscular component (tendons). Only in a particular joint position can the connective tissue transmit forces or signal in the sense of mechanoreceptor triggering $(+++$ versus $---)$. Blue: cartilage. 
FIGURE 6. (a) The alternative in-series organization of the iuxta-articular tissue. From inside to outside: articular capsule (blue); periarticular regular dense connective tissue (yellow) in series with periarticular muscle (red). (b) The alternative organization of iuxta- articular connective tissue organized in series to the muscular component. In all joint positions, the connective tissue of the joint is brought to tension, and is capable of transmitting forces and signaling in the sense of mechanoreceptor triggering (+++ and +++ . Blue: cartilage.

\section{ACKNOWLEDGMENT (addition)}

Figures 1 through 6 are used courtesy of Prof. H. van Mameren, Department of
Anatomy and Embryology, Maastricht University, The Netherlands, according to the technique as described in Reference \#9 (of the original article [van Mameren $\mathrm{H}$, Drukker J. A functional anatomical basis of injuries to the ligamentum and other soft tissues around the elbow joint: transmission of tensile and compressive loads. Int J Sports Med. 1984;5:88-92.]).

Corresponding author: Jaap van der Wal, MD, PhD, Department of Anatomy and Embryology (retired), Faculty of Health, Medicine and Life Sciences, University Maastricht, Minderbroedersberg 4-6, 6211 LK, Maastricht, The Netherlands

E-mail: jaap.vanderwal47@kpnmail.nl 